\title{
Leveraging Multiple Linear Regression for Wavelength Selection
}

Tony Lemos, John H. Kalivas Department of Chemistry

Idaho State University

921 S. $8^{\text {th }}$ Avenue, STOP 8023 Pocatello, ID 83209, USA lemoton2@isu.edu, kalijohn@isu.edu
Idaho State U N I V E R S I T Y
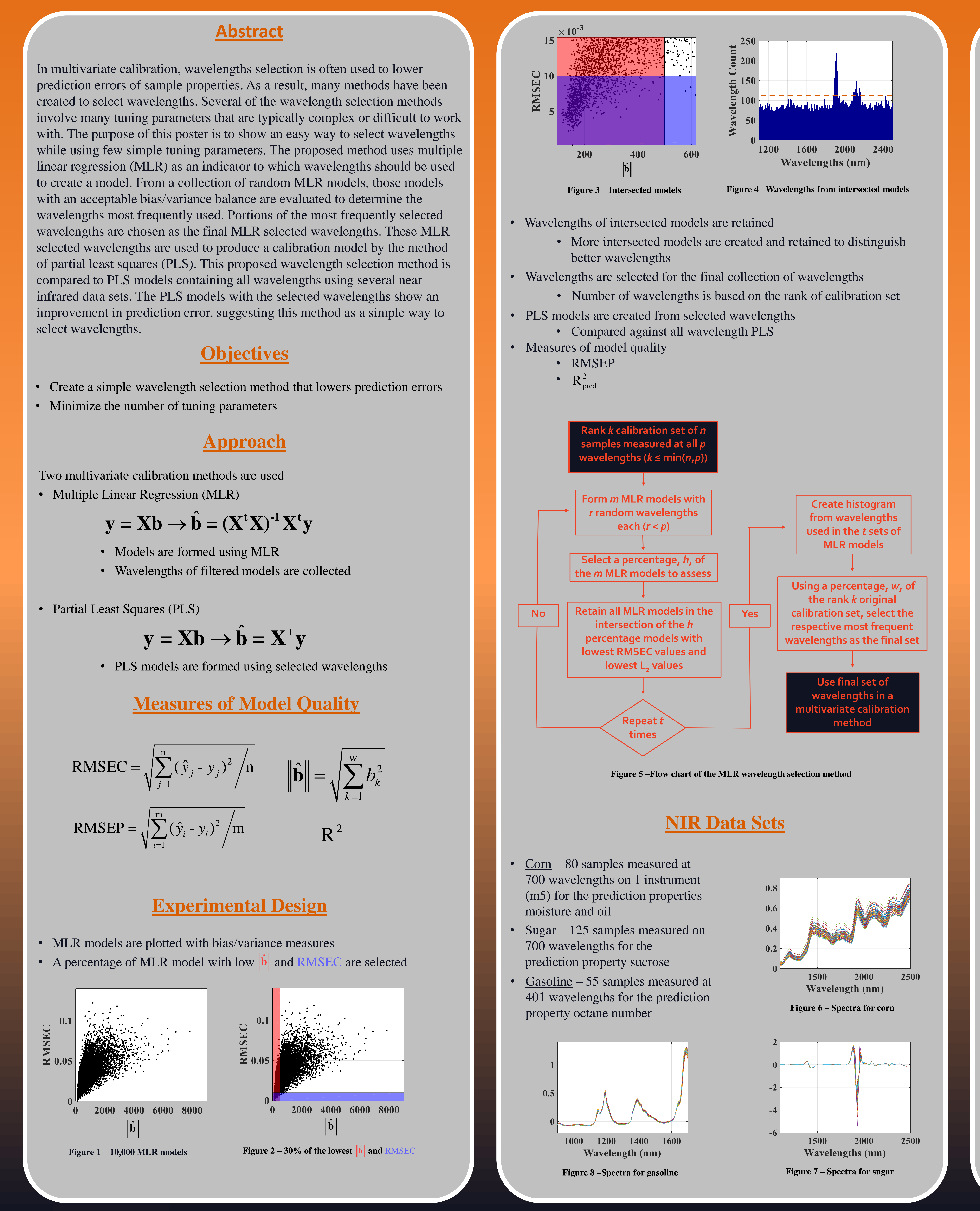

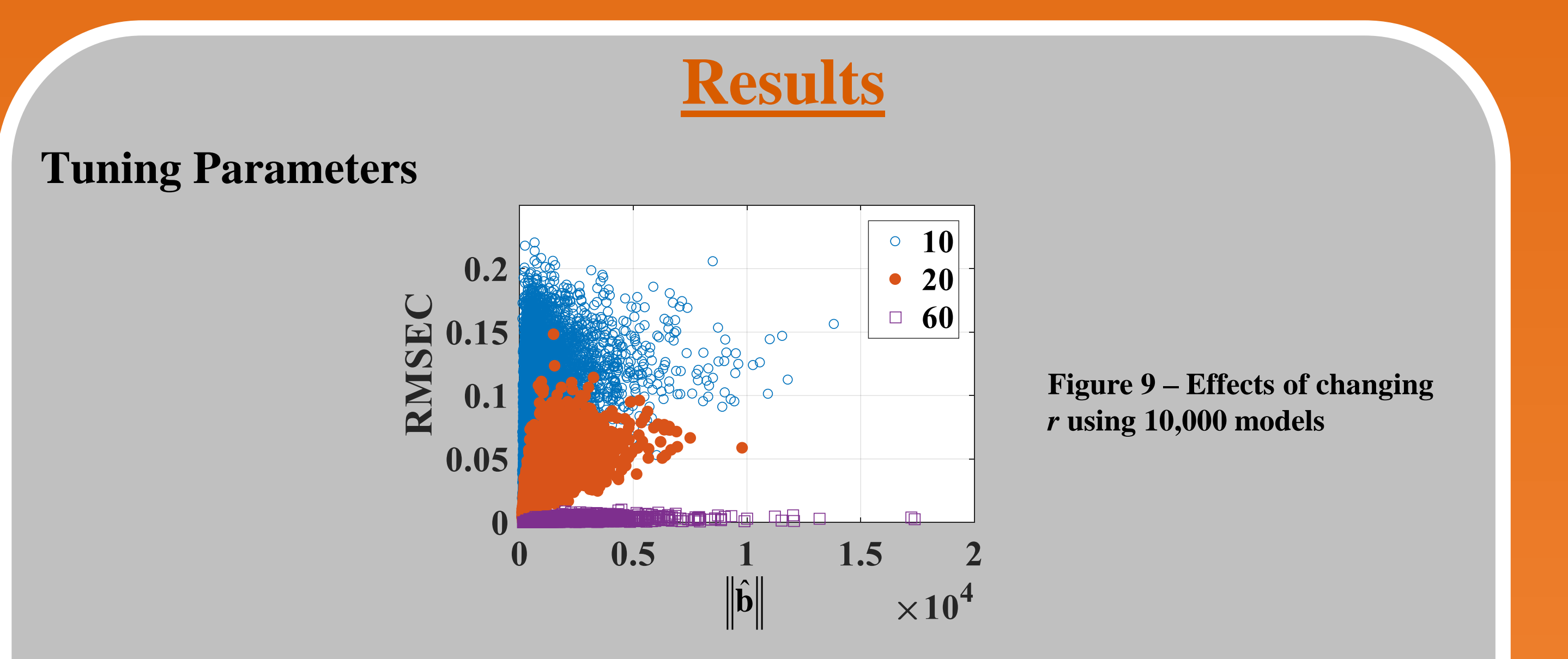

Number of wavelengths for each MLR model $(r)$
. Wavelengths will effect which models are intersected

More wavelengths, lower RMSEC

- Less wavelengths, lower $\mathrm{L}_{2}$ norm

For this study, $r$ is set to 20 wavelengths
nats

Number of MLR models $(m)$

- Small amounts do not show the range of MLR models

More models that are formed, the more likely the selected wavelength is useful
set to 10.000 models

Percentage of MLR models with low RMSEC and $\mathrm{L}_{2}$ norm $(h)$

over-fitter or under-fitted

- A large $h$ will allow poor models in the intersection

- A Small $h$ will not show which wavelengths are useful

The number of intersection sets $(t)$

- One inersection las nore

More intersection allows more dominant wavelengths to

appear more obvious

- The his

Percentage of selected wavelengths $(w)$

$w$ is based on the percentage of the rank
of the calibration set

A higher percentage, the mor
PLS model appear like all

wavelength PL

A lower percentage, worse the

PLS model performs
$0 \%$ is chosen for this stud $y$

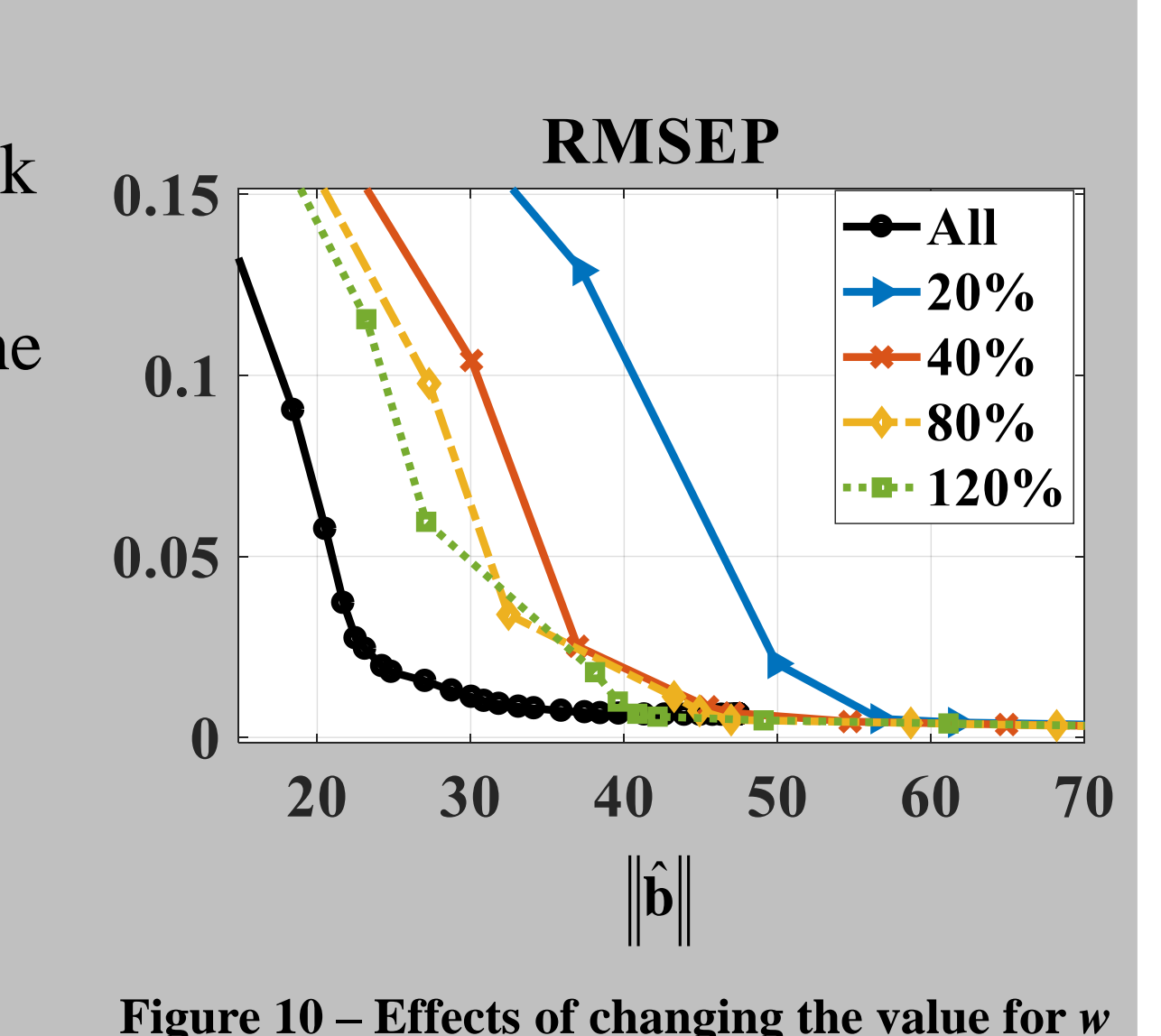

Corn - m5 Moisture


$\underset{\substack{\text { Figure } \\ \text { sucrose }}}{\substack{\text { sLS } \\ \text { mean results for sugar }}}$



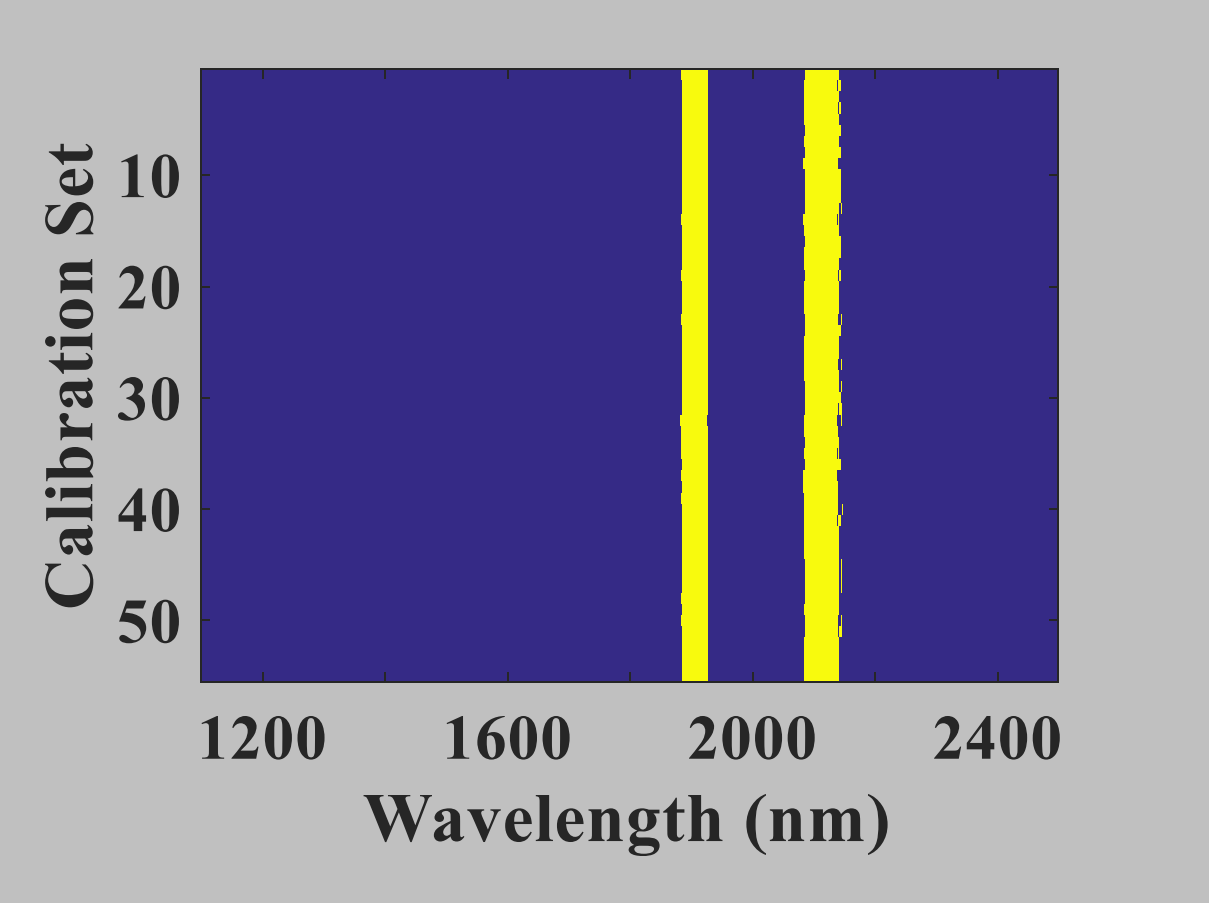

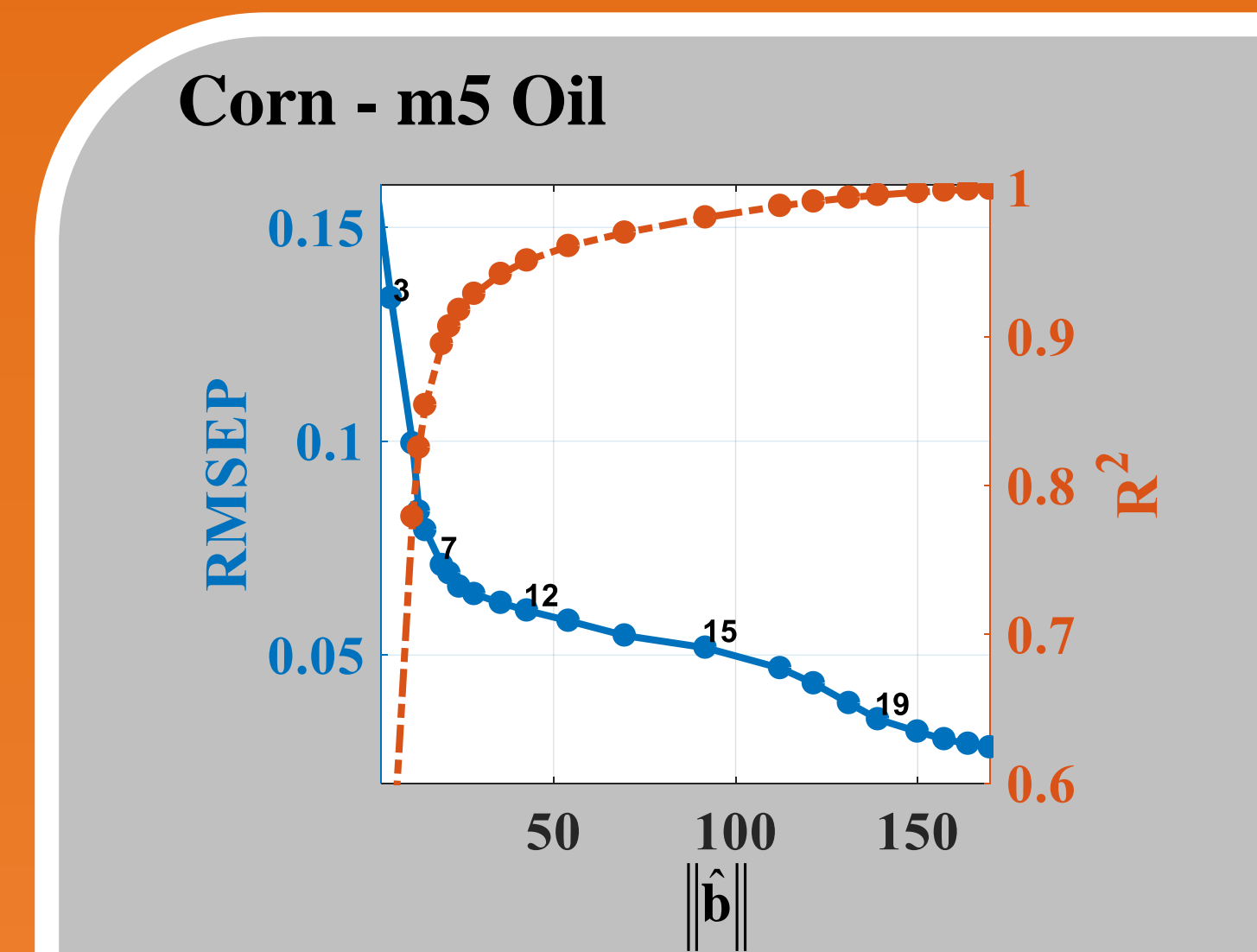


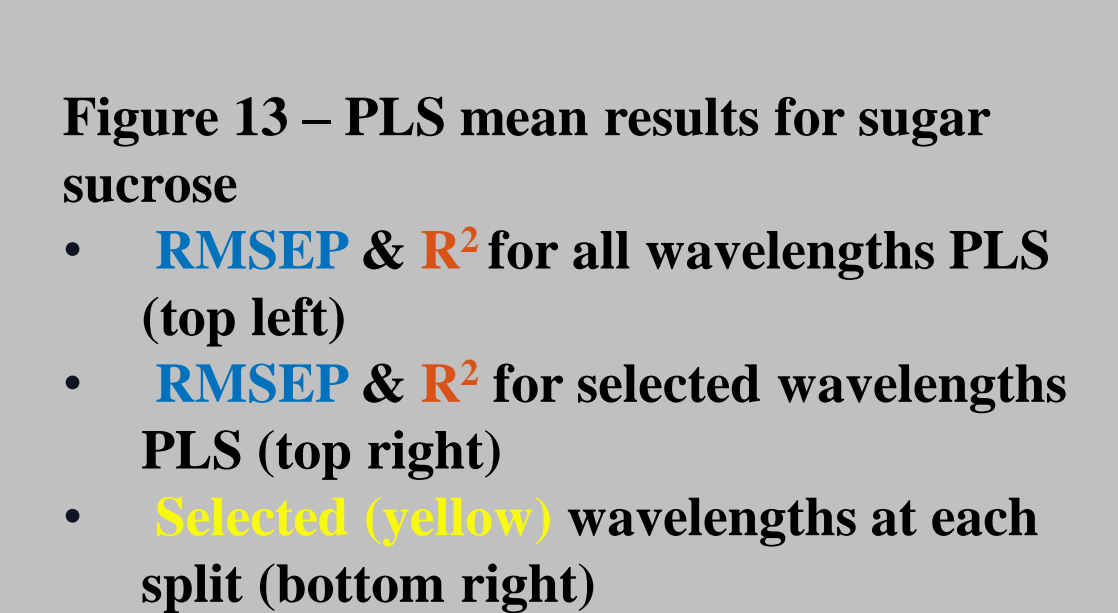

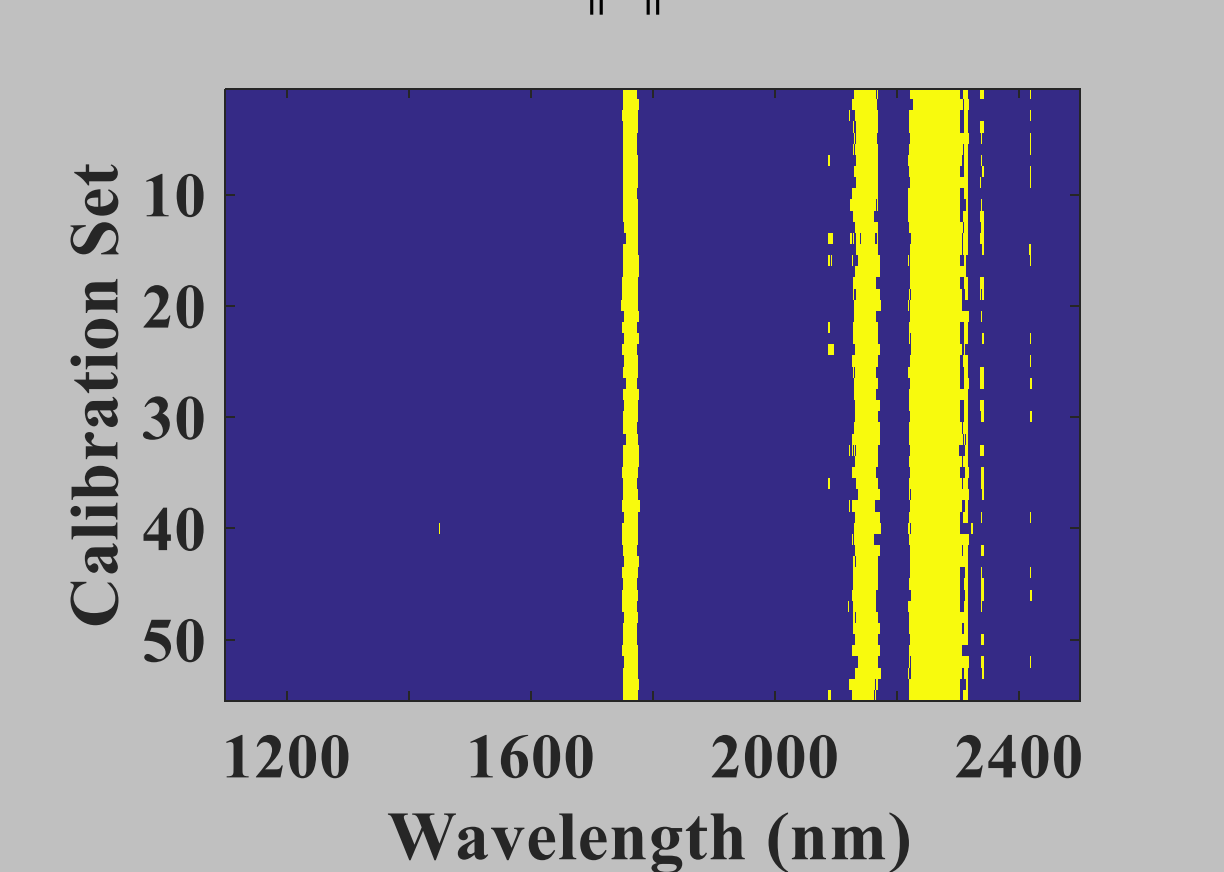





Conclusions



- Gengh selection helps from improved calibration models

- Generally does better than all wavelenght PLS

- Most datasets choses banded wavelengths

- Gasoline did

- Larger $\mathrm{L}_{2}$ norm

Tuning parameters
- Goal was
Oat

he number of parameters

Out of the five, only two can be changed

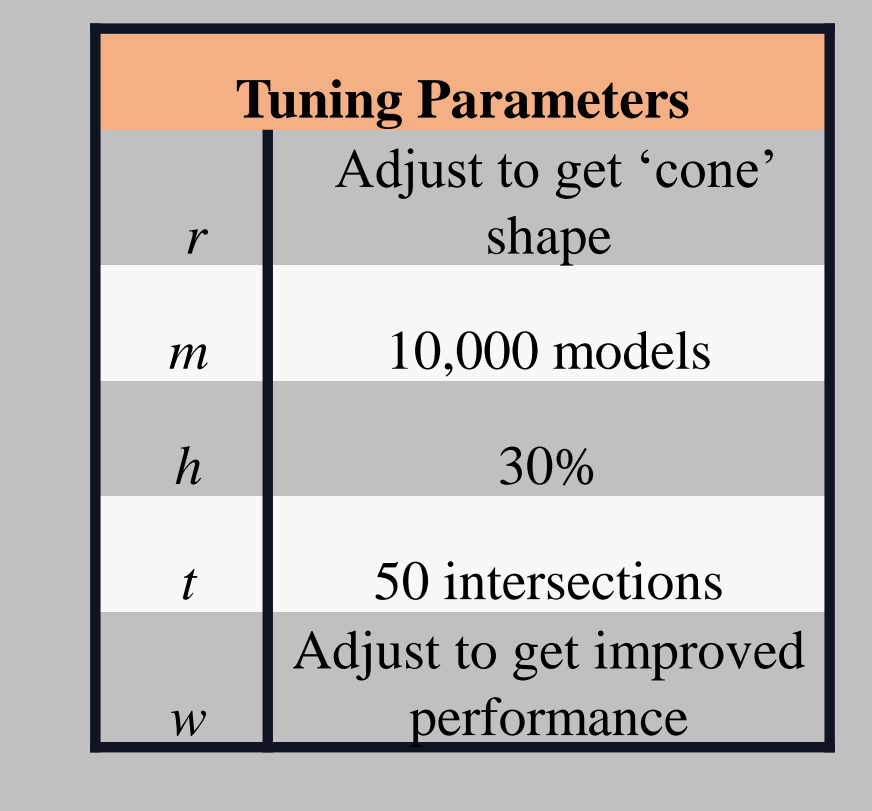

- The proposed methodis succesful and can be use fo

Acknowledgements: 